\title{
The Dosimetric Advantages of Robust Optimization Combined With Flatten Filter Free Mode in Left Breast Cancer
}

Hongying Luo

University of South China https://orcid.org/0000-0002-7688-8531

\section{Guoping Shan}

Zhejiang Cancer Hospital

\section{Kainan Shao}

Zhejiang Cancer Hospital

\section{Yiwei Yang}

Zhejiang Cancer Hospital

\section{Xia Zhou}

Zhejiang Cancer Hospital

Jianliang Zhou ( $\nabla$ jun_zhouliu@163.com )

University of South China

\section{Research}

Keywords: Robust optimization, Postoperative left breast cancer, Flatten filter free mode, Volumetric Modulated Arc Therapy, Dosimetric characteristics

Posted Date: November 13th, 2020

DOl: https://doi.org/10.21203/rs.3.rs-104605/v1

License: (a) This work is licensed under a Creative Commons Attribution 4.0 International License. Read Full License 


\section{Abstract}

Background and purposes

To explore the advantages of Robust optimization in dosimetry under flatten filter free(FFF) Mode after left breast cancer surgery.

Materials and methods

21 patients with left breast cancer after surgery were randomly selected from 2019 to 2020 . The planned target volume (PTV) dose was prescribed 50Gy /25times and B. With or without Robust optimization were designed on the RayStation planning system with Volume rotary intensity-modulated radiotherapy technology based on the FFF Mode. By moving the center point of the field to simulated the movement of target area of the internal chest wall $(0.50 \mathrm{~cm})$ caused by Respiratory movement, dosimetry characteristics. Using spss 23.0 to analyse the datum.

Results

when the chest wall target moved outward, the PTV target area $\mathrm{D}_{98}, \mathrm{D}_{95}, \mathrm{D}_{2}, \mathrm{Cl}$ and $\mathrm{HI}$ with Robust optimization were better than those without Robust, and the coverage rate of PTV-CHESTV 50 was significantly higher than that without Robust optimization $(P=0.000060)$, which was $15.39 \%$ higher than

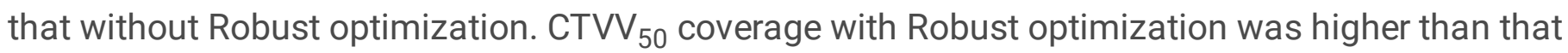
without Robust optimization, with an increase of $14.48 \%$. In terms of endangering organ parameters, the average spinal cord dose of the plan with Robust optimization was $13.19 \%$ lower than that of the plan without Robust optimization, and the lung $V_{5}$ of the plan without Robust optimization was slightly lower than that of the plan with Robust optimization, which was $1.80 \%$ lower than that of the plan without Robust optimization. There was no significant difference in machine execution efficiency between the two groups $(P>0.05)$.

\section{Conclusions}

The Robust optimization could be adopted in the development of postoperative left breast cancer radiotherapy plan, which ensures that the target dose coverage and the dose limit of organ-at-risk still meet the clinical requirements under condition of chest wall displacement caused by respiratory movement.

\section{Introduction}

Breast cancer is one of the main malignant tumors leading to female deaths worldwide, and its incidence has been increasing in recent years ${ }^{1}$. Radiation therapy of postoperative left breast cancer was suitable for patients with high risk of recurrence. Clinical Target Volume (CTV) was extremely complex, including high-risk recurrence areas and high-risk lymphatic drainage areas, especially including the chest wall, 
regional lymph nodes, supraclavicular area, axillary and internal mammary areas. Post-mastectomy radiotherapy (PMRT) in patients with breast cancer reduces the risk of locoregional recurrence (LRR). In addition, improvement in locoregional control can impact overall survival (OS) ${ }^{2}$.

Intensity-modulated Radiation Therapy(IMRT) and Volumetric Modulated Arc Therapy(VMAT) have been developed and used in breast cancer dosimetry research and clinical trials ${ }^{3-6}$. It has the advantage of optimizing plans to meet clinical goals, fast planning, treatment delivery, and more homogeneous target doses and tailored doses to organs at risk (OAR $)^{7-9}$, which even achieved better dosimetry results than Conventional Tangential Field Conformal Radiotherapy techniques ${ }^{10}$. Radiation induced heart diseases and cardiovascular events, which all have side effects caused by the definite effect of radiation. Intended to reduce patient's side effects, we introduced FFF Mode supported by Varian TrueBeam Accelerator (Varian Medical System, CA). The maximum dose rate of this mode is $1400 \mathrm{MU} / \mathrm{min}(6 \mathrm{MV})$, which is $7 / 3$ times highter than that of conventional Flatten Filter(FF) mode, and has better freedom of modulation in breast cancer radiotherapy plans by use partial arcs. FFF mode had less scatter than FF mode. At the same time, it greatly decreased patient's treatment time, reduced errors in respiratory movement, and lowered the probability of secondary malignancies ${ }^{11}$.In previous research, authors found that in the design of postoperative left breast radiotherapy plan, FFF mode was significantly superior to FF mode in the protection of ipsilateral lung, whole lung, heart, spinal cord and anterior descending coronary artery $(A D C A)^{12}$.

The main challenge in the process of providing breast cancer patients with VMAT treatment based on FFF mode(FFF-VMAT) was that when patient in respiratory movement, especially in the case of breast swelling or deformation during radiation therapy course, the treatment plan lacked of robustness. When breast cancer patients undergo radiotherapy, respiratory movement will raise the chest wall out to a certain distance. But margins of CTV is close to body surface, PTV cannot be extended to the outside of body surface ${ }^{13}$. In the use of Tangential Field Conformal Radiotherapy techniques, beams-eye-view (BEV) in multiple leaf Collimator (MLC) position could be edited to extend the irradiation range of beams in this direction outwardly, so as to ensure the dose near patient's epidermis could still meet clinical requirements. However, when using reverse optimization techniques such as VMAT for plan design, the MLC position could not be edited to realize the expansion of radiation field beyond skin of the chest wall target area, and there were certain risks of off-target. Usual solution was to add a certain thickness of Virtual Bolus (VB) on the target area's skin surface, and expanded PTV to VB. The optimized radiation therapy plan field could achieve a similar effect of expanding the tangent field's irradiation range. But in final dose assessment, VB should be removed and dose calculation should be performed again. The main disadvantage of this method was that dose distribution of radiotherapy plan used for the final evaluation was not optimized based on actual radiotherapy implementation status, so this plan was not guaranteed to be the actual optimal solution. Moreover, since VB removed away, the final dose distribution was inadequate for protection of organs at risk compared with the initial optimized results ${ }^{14}$. 
In the process of conventional radiotherapy plan design, considering the effects of intra-fraction errors and positioning errors, CTV is always needed to expand by a certain boundary to form Internal Clinical Volume (ITV) and PTV. In proton therapy, steep gradient of pencil beam made treatment extremely sensitive to uncertainty, and conventional extended boundary often failed to provide expected robustness to uncertainty. For Intensity Modulated Proton Therapy (IMPT), RayStation planning system (RaySearch Laboratories, Sweden) introduced Robust optimization tool in version 4.5.1 (Figure 3). Firstly, preset the possible moving direction and range of clinical target area, system will generate certain number of scenarios, traverse the value of the cost function in each scenario, and select the worst performing scenario as the next optimization benchmark. This method is called minimax optimization method ${ }^{15-16}$. It has been verified that the optimization tool can also be used to develop photon radiotherapy plans ${ }^{17-}$ 18. Using Robust optimization method in breast VMAT plan design can also achieve more robust optimization results than Tangential Field Conformal Radiotherapy and VB ${ }^{19-20}$.

When using FFF-VMAT technology to formulate a radiotherapy plans after postoperative left breast cancer, because of higher dose rate, it was necessary to carefully considered the impact of error caused by respiratory motion on the evaluation of planned dose. In this study, FFF-VMAT technology and Robust optimization method would be used to develop a modified radical mastectomy radiotherapy plan for left breast cancer. The conventional setup error was still processed by CTV external expansion to generate PTV, and the extra chest wall caused by respiratory movement used Robust tool for optimization. In the case that chest wall target area was shifted to a certain extent due to respiratory movement, by comparing the changes of target area coverage and organ dose at risk through the radiotherapy plan with or without Robust optimization, researchers evaluated the role of Robust optimization in FFF-VMAT radiotherapy design and provided reference method for treating postoperative left breast cancer patients.

\section{Materials And Methods}

A total of 22 female patients referred to cancer hospital Affiliated to the University of Chinese Academy of Sciences with modified radical mastectomy were recruited during the period from January 2019 to February 2020. In order to reduce the differences in delineation of target areas and organs at risk, all patients were selected from the same chief physician, which has the median age of 56 years old $(33,78)$. Patients were scanned with Phillips Brilliance large aperture CT (Philips, BrillianceTM Big Bore $\mathrm{CT}$,Netherlands) with 5-mm scanning interval, and the scanning range was from the angle of Mandible to Navel. After getting scanned, the CT images were transmitted to RayStation planning system. Then the doctor would sketch target area and OARs according to 2020 National Comprehensive Cancer Network(NCCN).

\section{Target and normal tissue delineation}

The CTV and heart were delineated by a radiation oncologist, in the same time contoured the lungs, spinal canal, contralateral breast and external contour. The CTV included the left breast/chest wall and the supraclavicular and axillary level I-III nodes. All structures were delineated according to published international guidelines. PTV was automatically generated, derived from CTV with $5 \mathrm{~mm}$ extension in the 
superior-inferior/anterior-posterior/left-right directions. The same experienced clinician drew targets and OARs based on scanned transmitted CT images and clinical NCCN guidelines, including PTV that was CTV enlarged 5mm in all direction; CTV-CHEST: chest wall; CTV-IMM: internal mammary area; PTVCHEST:CTV-CHEST enlarged 5mm in all direction; PTV-IMM: CTV-IMM enlarged $5 \mathrm{~mm}$ in all direction; CTV ALN: axillary region; SC: supraclavicular region, including I, II, III; OARs included ipsilateral lung, right lung, heart, spinal cord. Completed clinical targets and OARs were in need of the review by the chief physician.

\section{Plan Design}

All patients were unified by a same physicist using the RayStation planning system for radiotherapy planning. The X-ray of $6 \mathrm{MV}$ was selected in plan, and the clinical prescription dose in planned target area was $50 \mathrm{~Gy} / 25$ fractions. Intending to ensure radiation dose on the surface of breast skin, a $0.50 \mathrm{~cm}$ compensation film made of a material similar to water was added to treatment planning and actual treatment process. Treatment plan was designed on RayStation planning system. Each patient took the same prescription dose at the same CT and the same clinical target area to design two groups of FFFVMAT plans with or without Robust optimization with unified final dose to $95 \%$ of PTV volume covered by prescription dose $\left(\mathrm{D}_{95}\right.$ was equal to prescription dose $5000 \mathrm{cGy}$ of the planned target). In order to counteract possible outward movement of partial target area of chest wall(PTV-CHEST) caused by respiratory movement, when formulating Robust optimization plan, the minimum dose-volume histogram(DVH) objective function of PTV-CHEST was set to "Robustness". "Robustness" option was set to Anterior $1.00 \mathrm{~cm}$, Left $1.00 \mathrm{~cm}$, other directions default to $0 \mathrm{~cm}$. Robustness optimization was carried out for two directions in which chest wall target might move outwardly (to prevent miss). System would generate five optimization scenarios for comprehensive evaluation, which combined into the final optimization solutions ${ }^{19}$.

\section{Plan analysis}

First of all, comparative statistics of dosimetric parameters of target and endangered organs were carried out for each patient with or without Robust optimization. Then the dose distribution generated from the original plan was carried out by simultaneously moving $X$ and $Y$ coordinates of center of original planned radiation field without changing other planning parameters so as to simulate outward movement of target caused by human respiratory movement. Dosimetric parameters of the target and OARs of two groups of plans with or without Robust optimization were compared and evaluated to each other.

Next is the data to be collected for evaluation and analysis $\llbracket$

(1) Evaluation of planned target area: it was required to meet the clinical prescription dose. Statistics before and after shifting of planned target PTV $D_{98}, D_{95}, D_{2}\left(D_{x}\right.$ represented dose of $x \% P T V$ volume irradiated), conformality index $(\mathrm{Cl})$, Heterogeneity Index $(\mathrm{HI})$. Among them, $\mathrm{Cl}=\left(\mathrm{PTV} \mathrm{V}_{\text {ref }} / \mathrm{V}_{\mathrm{PTV}}\right) /\left(\mathrm{PTV}_{\text {ref }} / \mathrm{V}_{\text {ref }}\right)$, PTV $V_{\text {ref }}$ is prescription dose of PTV, $V_{\text {PTV }}$ is volume of PTV, and $V_{\text {ref }}$ is volume of prescription dose accepted by whole body $\left(V_{x}\right.$ represented percentage of target volume containing $x G y, D_{\max }$ represented maximum dose of target). $\mathrm{Cl}$ value is between 0 and 1 . The closer to 1 , the better is the fitness of the 
target area. $H I=\left(D_{2}-D_{98}\right) / D_{50}, H I$ value is between 0 and 1 , the closer to 0 , the better the uniformity.

(2) Dose parameters of OARs: before and after shifting, $V_{30}, V_{20}, V_{5}$ Mean Dose $\left(D_{\text {mean }}\right)$ of ipsilateral lung and whole lung, $V_{5}$ and $D_{\text {mean }}$ of right lung, Heart's $V_{40}$ and $D_{\text {mean }}, V_{0.1}\left(\mathrm{~cm}^{3}\right)\left(V_{x}\left(\mathrm{~cm}^{3}\right)\right.$ of brachial plexus represented the maximum dose in $\mathrm{xcm}^{3}$ volume ) and $\mathrm{V}_{0.3}\left(\mathrm{~cm}^{3}\right)$ dose of anterior descending coronary artery need to be collected.

(3) Statistical methods: the data obtained were statistically analyzed by SPSS 23.0 software, and the Wilcoxon signed-rank was carried out for the results of FFF-VMAT plans with or without Robust optimization. The quantitative parameters were expressed as mean \pm variance $(0$, and the two significant digits after the decimal point were retained (when $P<0.05$, it was considered a statistical difference existed).

\section{Results}

\section{Comparison of target dose parameters}

According to Fig.1, before getting shifted, the Cl of PTV with Robust optimization were lower than those without Robust optimization $(P<0.05)$. PTV-CHEST with Robust optimization was slightly better than that without Robust optimization $(P=0.000060)$. There were no statistical differences in other parameters. Although there were slight differences in some dosimetric parameters between two groups, they all met requirements of clinicians.

In order to simulate movement of the chest wall caused by respiratory movement, the dose distribution with or without Robust optimization plan was calculated and evaluated after the field center was shifted to $0.50 \mathrm{~cm}$. The $D_{98}, D_{95}, D_{2}$ and Cl of PTV with Robust optimization were higher than those without Robust optimization. HI of PTV was lower than that without Robust optimization(Fig.2). $V_{50}$ of PTVCHEST was higher than that without Robust optimization. CTV was higher than that without Robust optimization ( $P=0.0039)$. Fig. 3 and Fig. 4 could strongly prove this result. The difference of CTV coverage before and after Robust optimization or without Robust optimization could be found. The blade width of planned multi-leaf collimator with Robust optimization was about $0.80 \mathrm{~cm}$ larger than that without Robust optimization(Fig. 5).

\section{Comparison of OARs dose parameters}

Before displacement, the ipsilateral lung's $V_{30}$, whole lung's $V_{30}$, heart's $D_{\text {mean }}$, heart's $V_{40}$, anterior descending coronary artery and spinal cord with Robust optimization were significantly lower than treatment plan without Robust optimization, there was no statistical difference in other parameters(Fig.2). After transposition, $\mathrm{V}_{5}$ of lung without Robust optimization was significantly lower than that of treatment plan with Robust, $D_{\max }$ of spinal cord of treatment plan with Robust optimization was significantly lower than that of treatment plan without Robust optimization ( $P=0.000026)$. There were no statistical differences in other dose parameters. After evaluation, dose of endangering organs met clinical requirements. 


\section{Discussion}

In this study, we aimed to solve the problem of insufficient clinical target dose due to respiratory motion during radiotherapy for postoperative left breast cancer patients. Robust optimization module was introduced to limit the minimum dose of PTV-CHEST in patient's planned target area. After shifting, we found that the Robust optimization plans provided better target coverage, $H I, P T V D_{98}, D_{95}$ and $D_{2}$ than those without Robust optimization plans $(P<0.05)$. The dose distribution in these Robust optimization plans was less affected by perturbations. The PTV-CHEST with the greatest activity had Robust optimization slightly better than Robust optimization $(P=0.000060)$. The target coverage rate of $C T V V_{50}$ without Robust optimization was $79.12 \pm 9.51 \%$, and the target coverage rate with Robust optimization was $90.58 \pm 4.42 \%$. There was a significant difference in the target coverage of $C T V V_{50}$ between the two treatment plans $(P=0.000069, P<0.05)$. It could be clearly seen from Fig. 3 that shifted center of field leads to changes in CTV coverage.

In terms of OARs, it was mainly statistically different between $V_{5}$ of patients' ipsilateral lung and $D_{\max }$ of spinal cord. The treatment plan of $\mathrm{V}_{5}$ without Robust optimization in patients' ipsilateral lung was lower than that with Robust optimization, and $D_{\max }$ of spinal cord was significantly higher than those were enrolled in Robust optimization. In terms of machine execution efficiency, there was no statistical difference between them in terms of total beam time and total MU.

In order to reduce the errors caused by patients' respiratory movement in actual treatment, the Robust optimization technology was added to radiotherapy plan, and clinical studies had shown that it could not only improve the coverage rate of the clinical target area, but also reduce the dose of OARs. The Mahmoudzadeh team's experiment proved that the introduction of Robust optimization could potentially reduce the need for deep inspiration breath-hold technology, allowing patients to reduce the exposure dose to heart with breathe freely, and improve the dose coverage of tumor area ${ }^{21}$. Fredriksson proposed that adding Robust optimization could significantly increase the patient's skin dose, and to some extent, it could replace the efficacy of VB ${ }^{22}$. The Alex Dunlop team added Robust optimization to study breast cancer radiotherapy based on organ motion, found that $\mathrm{D}_{98}, \mathrm{D}_{95}, \mathrm{D}_{50}$ and $\mathrm{D}_{2}$ of CTV that added Robust optimization were significantly different from treatments without Robust optimization $(P<0.01)$, they proposed that the use of robust optimization based on organ motion to generate VMAT plan is clinically acceptable for the typical and extreme target area changes during treatment ${ }^{14}$. Hideharu Miura et al. verified the advantages of introducing Robust in other tumor types with greater CTV activity, such as Larynx cancer,Robust-optimized plan had better CTV coverage rate than that without Robust-optimized, and less carotid artery exposure dose ${ }^{23}$. This was slightly different from actual exposure dose of OARs dose parameters in this study, the exposure dose of ipsilateral lung V5 was higher than that without Robust optimization, because the improved CTV coverage in our study was at the expense of a low dose bath to healthy tissue, thereby delivering a higher volume of low dose to the ipsilateral lungs than that without Robust optimization plans. 
Designed PTV was aim to reduce the error impact of a series of radiotherapy procedures on CTV, while the clinical target area of postoperative left breast cancer patient is long and narrow, and close to the surface of skin, CTV can only expand to the chest cavity, but not to the outer surface of skin, compared with other tumor target areas, respiratory movement is more likely to affect actual radiation dose of breast cancer's CTV, introduced of Robust optimization method significantly improved this situation.As could be seen from Figure 4, the blade width of multi-blade collimator in the Robust optimization plan increased by about $0.81 \mathrm{~cm}$ compared with that without Robust optimization. When the center of field moved a same distance, the coverage rate of $\mathrm{CTVV}_{50}$ dose with Robust optimized treatment plan increased by about $14.49 \%$, while $V_{5}$ of the ipsilateral lung only increased by about $1.00 \%$,the reason for the increase was the introduction of Robust optimization, which optimized the movement of target area to the left and front due to respiratory movement, when seeking the best solution under the condition of increasing uncertain factors, the actual volume of the illuminated target area increases and the scattering amount increased in the same proportion. Although Robust optimization could ensure the dose coverage of target area to a certain extent, it was still recommended if patient's respiratory movement amplitude caused the difference between outer contour of target area and the planned target area to exceed $0.50 \mathrm{~cm}$ during CBCT verification before implemented the treatment plan, re-check the patient's position or use deep inspiration breath-hold techniques as appropriate.

In summary, under the premise that with or without Robust optimization met clinical requirements before displacement, the deviation of CTV target area of human respiratory motion was simulated by changing the position of center point of radiation field. VMAT plans using the robustness feature of RayStation are less affected in average than that without Robust optimization, it could be seen after the shift that the treatment plan optimized by Robust was better for the $\mathrm{D}_{98}, \mathrm{D}_{95}, \mathrm{D}_{2}, \mathrm{Cl}, \mathrm{HI}$ of PTV and the most active PTVCHEST dose parameters, and in most important CTV coverage rate, the Robust optimized plan was significantly higher than the without Robust optimization plan, meanwhile better CTV prescription dose coverage usually means more effective tumor control rates. Therefore, FFF-VMAT Robust optimization treatment plan is better than the plan without Robust optimization. If more Robust is needed to optimize the follow-up radiotherapy effect and evidence of clinical use, the sample size can be increased for research.

\section{Conclusions}

The Robust optimization could be adopted in the development of postoperative left breast cancer radiotherapy plan, which ensures that the target dose coverage and the dose limit of organ-at-risk still meet the clinical requirements under condition of chest wall displacement caused by respiratory movement.

\section{Abbreviations}

FFF: Flatten filter free 
PTV: Planned target volume

CTV: Clinical target volume

PMRT: Post-mastectomy radiotherapy

LRR: Locoregional recurrence

OS: Overall survival

IMRT: Intensity-modulated radiation therapy

VMAT: Volumetric modulated arc therapy

OAR: Organs at risk

FF: Flatten filter

ADCA: Anterior descending coronary artery

FFF-VMAT $\triangle$ VMAT treatment based on FFF mode

BEV: Beams-eye-view

MLC: Multiple leaf collimator

VB: Virtual bolus

ITV: Internal clinical volume

NNCN: National comprehensive cancer network

DVH: Dose-volume histogram

\section{Declarations}

Ethics approval and consent to participate

Not applicable

Consent for publication

Not applicable

\section{Availability of data and materials}


The datasets used and/or analysed during the current study are available from the corresponding author on reasonable request.

\section{Competing interests}

The authors declare that they have no competing interests.

\section{Funding}

This study was supported by grants from Health Department of Zhejiang Province(2019C03003ロ 2020KY472).

\section{Authors' contributions}

LHY participated in the design of the study, performed the experiments and the statistical analysis, and drafted the manuscript. SKN performed the experiments and the statistical analysis, drafted the manuscript, and assisted with the manuscript preparation. YYW drafted the manuscript and assisted with the manuscript preparation. All authors read and approved the final manuscript.

\section{Acknowledgements}

Not applicable.

\section{References}

1. Ferlay J, Shin H R, Bray F, et al. Cancer incidence and mortality worldwide[J]. Lyon: International Agency for Research on Cancer, 2010.

2. Thorsen $L$ B J, Offersen B V, Dan $\varnothing \mathrm{H}$, et al. DBCG-IMN: a population-based cohort study on the effect of internal mammary node irradiation in early node-positive breast cancer. Journal of Clinical Oncology, 2016, 34(4): 314-320.

3. Lin Y, Wang B,et al. Dosimetric absorption of intensity-modulated radiotherapy compared with conventional radiotherapy in breast-conserving surgery. Oncology letters, 2014, 9(1): 9-14.

4. Dogan N, Cuttino L, Lloyd R, et al. Optimized dose coverage of regional lymph nodes in breast cancer: the role of intensity-modulated radiotherapy. International Journal of Radiation Oncology Biology Physics, 2007, 68(4): 1238-1250.

5. Donovan E, Bleakley N, Denholm E, et al. Randomised trial of standard 2D radiotherapy (RT) versus intensity modulated radiotherapy (IMRT) in patients prescribed breast radiotherapy. Radiotherapy and Oncology, 2007, 82(3): 254-264.

6. Pignol J P, Olivotto I, Rakovitch E, et al. A multicenter randomized trial of breast intensity-modulated radiation therapy to reduce acute radiation dermatitis. Journal of Clinical Oncology, 2008, 26(13): 2085-2092. 
7. Dogan N, Cuttino L, Lloyd R, et al. Optimized dose coverage of regional lymph nodes in breast cancer: the role of intensity-modulated radiotherapy. International Journal of Radiation Oncology Biology Physics, 2007, 68(4): 1238-1250.

8. Viren T, Heikkilä J, Myllyoja K, Koskela K, Lahtinen T, Seppälä J. Tangential volumetric modulated arc therapy technique for left-sided breast cancer radiotherapy. Radiat Oncol 2015:10-79.

9. Chen Y Z, Li J, Liao X F. Dosimetric comparison between VMAT and IMRT for postoperative radiotherapy of breast carcinoma. Journal of Cancer Control and Treatment, 2014, 27(5): 226-230.

10. Ranger A, Dunlop A, Hutchinson K, Convery H, Maclennan MK, Chantler $\mathrm{H}$,et al. A dosimetric comparison of breast radiotherapy techniques to treat locoregional lymph nodes including the internal mammary chain. Clin Oncol 2018;30(6):346-53.

11. Hall E J . Intensity-modulated radiation therapy, protons, and the risk of second cancers. International Journal of Radiation Oncologybiologyphysics, 2006, 65(1):1-7.

12. Luo HY®Zhou JL『Shan GP,et al..Analysis of dosimetric advantages of flatten filter free mode in postoperative left breast cancer.Cinese Journal of medical Physics $₫ 2020$ WHave been hired.

13. Sankar A, Velmurugan J. Different intensity extension methods and their impact on entrance dose in breast radiotherapy: a study[J]. Journal of Medical Physics/Association of Medical Physicists of India, 2009, 34(4).

14. Dunlop A, Colgan R, Kirby A, et al. Evaluation of organ motion-based robust optimisation for VMAT planning for breast and internal mammary chain radiotherapy. Clinical and translational radiation oncology, 2019, 16: 60-66.

15. Fredriksson A, Forsgren A, Hårdemark B. Minimax optimization for handling range and setup uncertainties in proton therapy. Medical physics, 2011, 38(3): 1672-1684.

16. Liu W, Zhang X, Li Y, et al. Robust optimization of intensity modulated proton therapy. Medical physics, 2012, 39(2): 1079-1091.

17. Stuschke M, Kaiser A, Pöttgen C, et al. Potentials of robust intensity modulated scanning proton plans for locally advanced lung cancer in comparison to intensity modulated photon plans. Radiotherapy and Oncology, 2012, 104(1): 45-51.

18. Stuschke M, Kaiser A, Jawad J A, et al. Multi-scenario based robust intensity-modulated proton therapy (IMPT) plans can account for set-up errors more effectively in terms of normal tissue sparing than planning target volume (PTV) based intensity-modulated photon plans in the head and neck region. Radiation Oncology, 2013, 8(1): 1-5.

19. Christer Andre Jensen, Ana María Acosta Roa, Marie Johansen,et al.Robustness of VMAT and 3DCRT plans toward setup errors in radiation therapy of locally advanced left-sided breast cancer with DIBH.Physica Medica,2018:12-18.

20. Nguryen $D$, Corbet $C$, Largeron $G$, et al. Is Robust optimization better than virtual bolus method to achieve skin flash in breast VMAT plans?Radiotherapy and Oncology. 2018, 127: S1027-S1027.

21. Mahmoudzadeh H, Lee J , Chan T C Y , et al. Robust optimization methods for cardiac sparing in tangential breast IMRT. Medical Physics, 2015, 42(5):2212-2222. 
22. Fredriksson,Hårdemark.Skin flash of breast in IMRT and VMAT using Robust optimization.Radiotherapy and Oncology,2012:S575-S576.

23. Miura H, Doi $Y$, Ozawa $S$, et al. Volumetric modulated arc therapy with robust optimization for larynx cancer. Physica Medica, 2019, 58: 54-58.

24. Xiaoying Liang,Raymond B.Mailhot Vega,Zuofeng Li,et al.Dosimetric consequences of image guidance techniques on Robust optimized intensity-modulated proton therapy for treatment of breast Cancer. Radiation oncology,2020, Vol.15 (99), 987-998.

25. van der Veen $\mathrm{G} J$, Janssen $\mathrm{T}$, Duijn A, et al. A robust volumetric arc therapy planning approach for breast cancer involving the axillary nodes[J]. Medical Dosimetry, 2019, 44(2): 183-189.

\section{Figures}




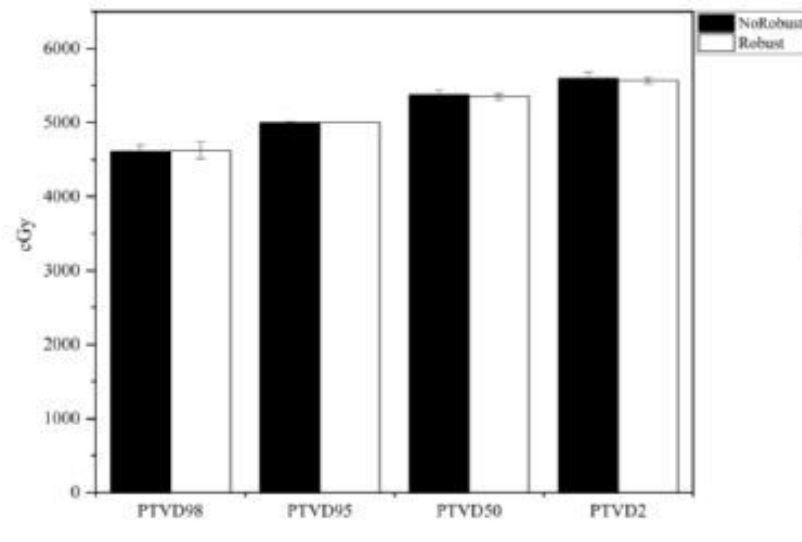

(A)

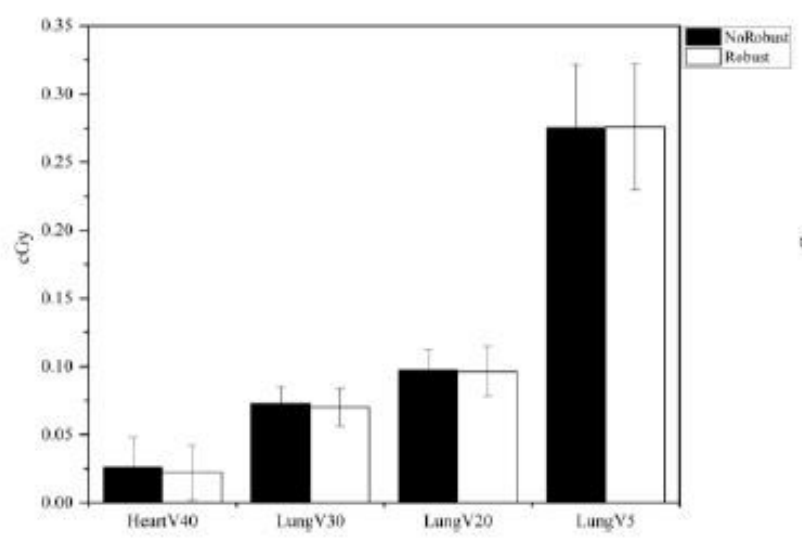

(C)

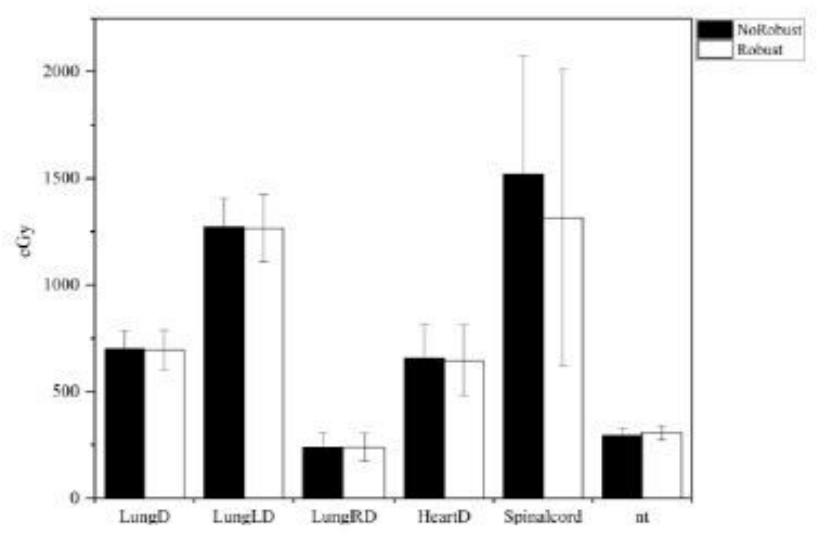

$(\mathrm{E})$

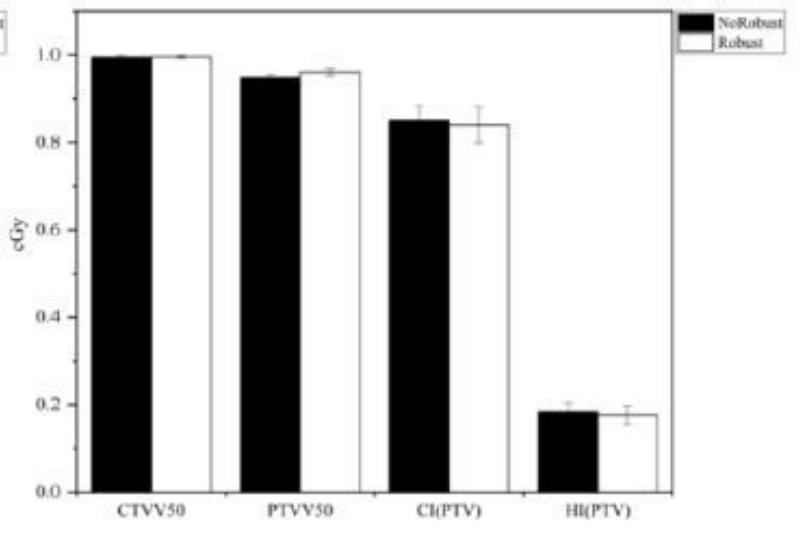

(B)

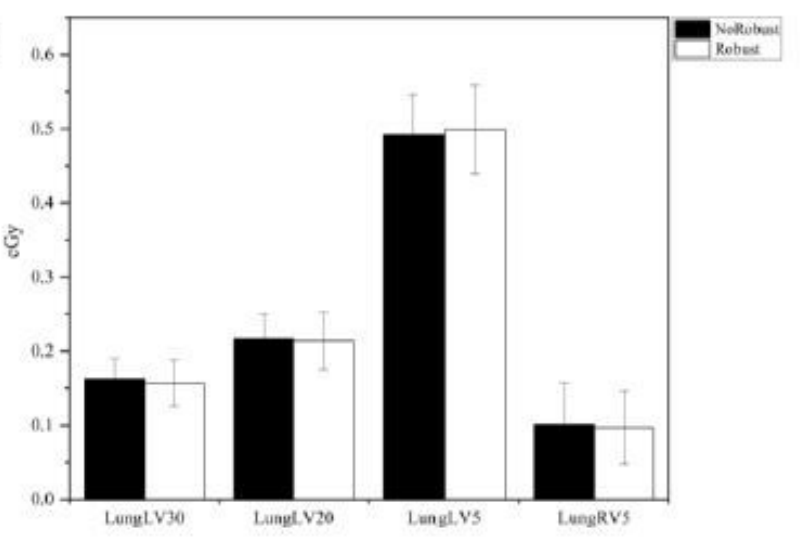

(D)

\section{Figure 1}

Dosimetry comparison of Target areas, OARs and Normal Tissue before displacement of central point of shooting field between No-Robust and Robust mode plans. $P(P T V D 98)=0.82 ; P(P T V D 95)=0.12$; $P(P T V D 50)=0.17 ; P(P T V D 2)=0.085 ; P(C T V V 50)=0.16 ; P(P T V V 50)=0.000060 ; P(C l)=0.025 ; P(H I)=0.27 ;$ $P($ HeartV40 $)=0.00014 ; P($ LungV30 $)=0.0021 ; P($ LungV20 $)=0.50 ; P($ LungV5) $=0.74 ; P($ LungLV30 $)=0.0021 ;$ 
$P($ LungLV20) $=0.48 ; P($ LungLV5) $=0.010 ; P($ LungRV5) $=0.19 ; P($ Lung $)=0.48 ; P($ LungLD $)=0.85 ;$ $P($ LungRD $)=0.99 ; P($ HeartD $)=0.092 ; P($ SpinalcordD2 $)=0.0037 ; P(n t D)=0.00042$.

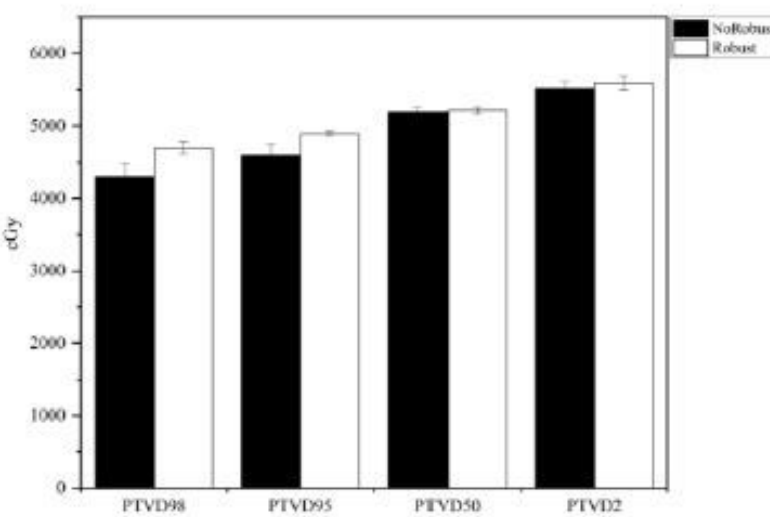

(A)

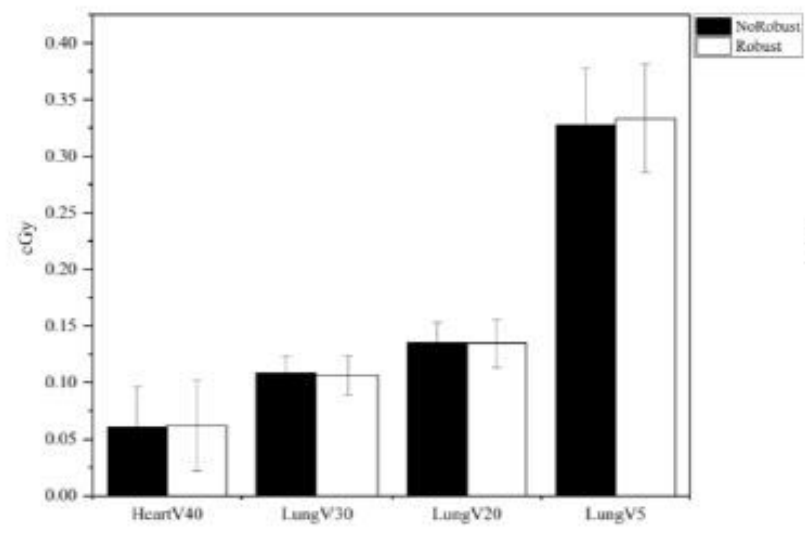

(C)

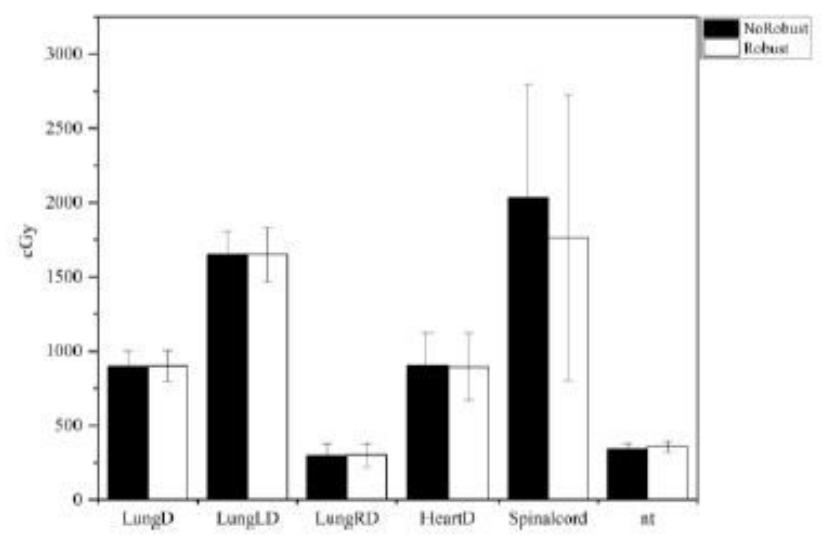

$(\mathrm{E})$

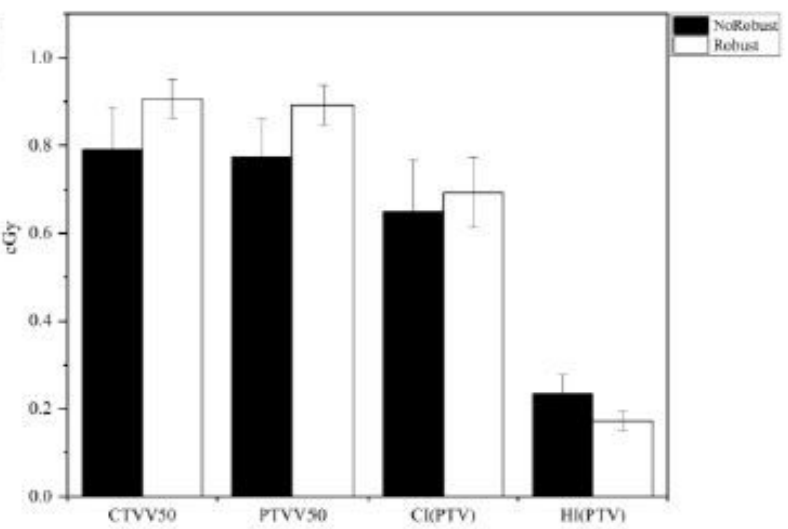

(B)

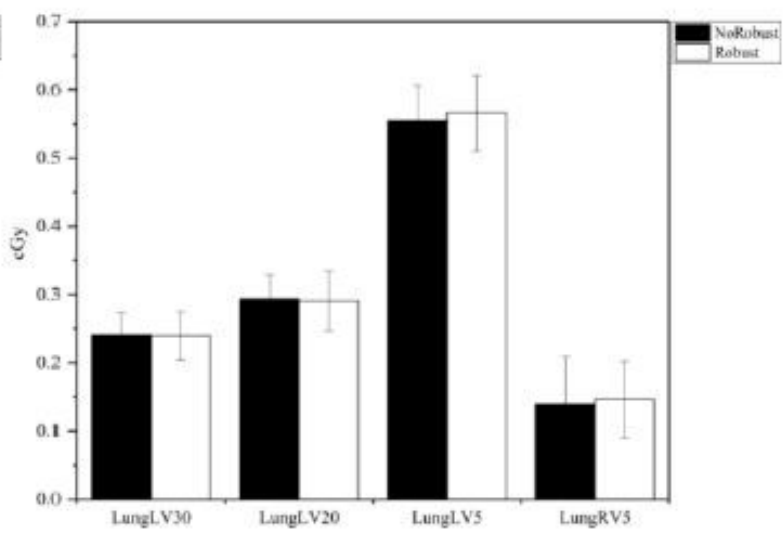

(D)

\section{Figure 2}

Comparison of dose of Target areas,OARs and Normal Tissue after displacement of central point of shooting field between no-Robust and Robust mode plans. Figure.1 Dosimetry comparison of Target areas,OARs and Normal Tissue before displacement of central point of shooting field between No-Robust 
and Robust mode plans. $P(P T V D 98)=0.000060 ; P(P T V D 95)=0.000060 ; P(P T V D 50)=0.092$;

$P(P T V D 2)=0.00062 ; P(C T V V 50)=0.000069 ; P(P T V V 50)=0.000060 ; P(C l)=0.00082 ; P(H I)=0.00011$; $P($ HeartV40 $)=0.010 ; P($ LungV30 $)=0.14 ; P($ LungV20 $)=0.48 ; P($ LungV5) $=0.058 ; P($ LungLV30 $)=0.21$; $P($ LungLV20) $=0.74 ; P($ LungLV5) $=0.0012 ; P($ LungRV5) $=0.99 ; P($ LungD $)=0.18 ; P($ LungLD $)=0.068$; $P($ LungRD $)=0.26 ; P($ HeartD $)=0.14 ; P($ SpinalcordD2 $)=0.0015 ; P(n t D)=0.00019$.

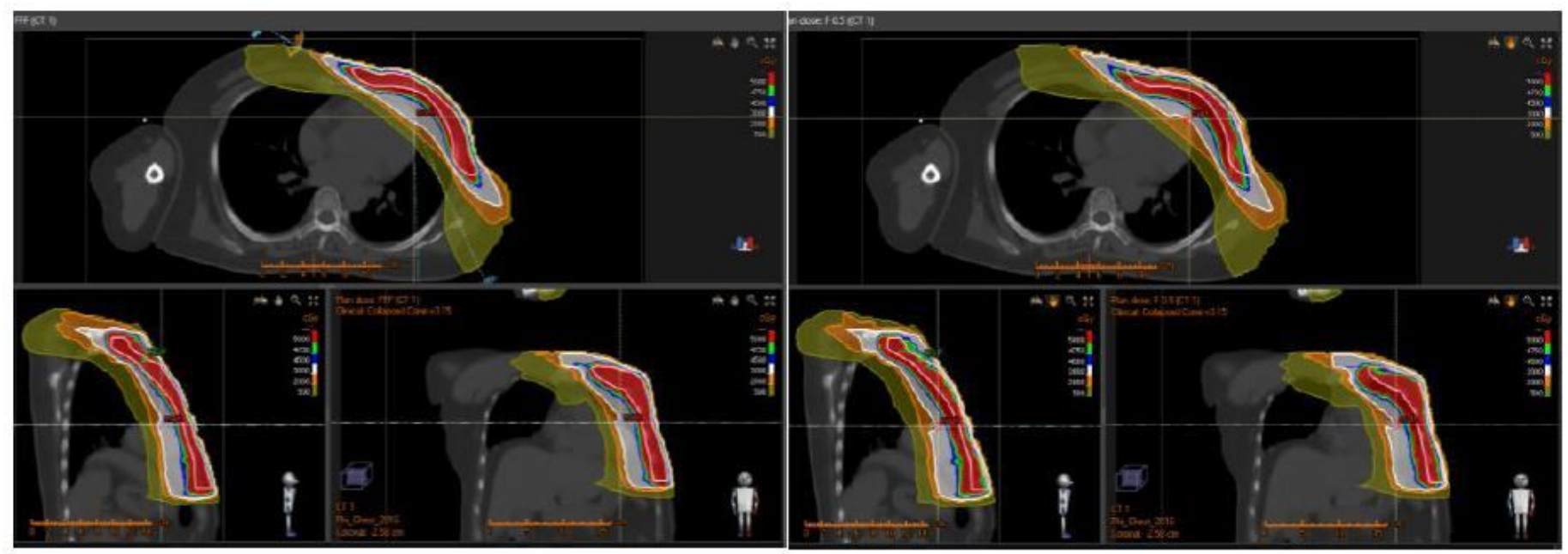

(A)

(B)

\section{Figure 3}

Dose distribution of CTV before and after displacement of central point of field without Robust optimization(A: before shifting;B :after shifting)

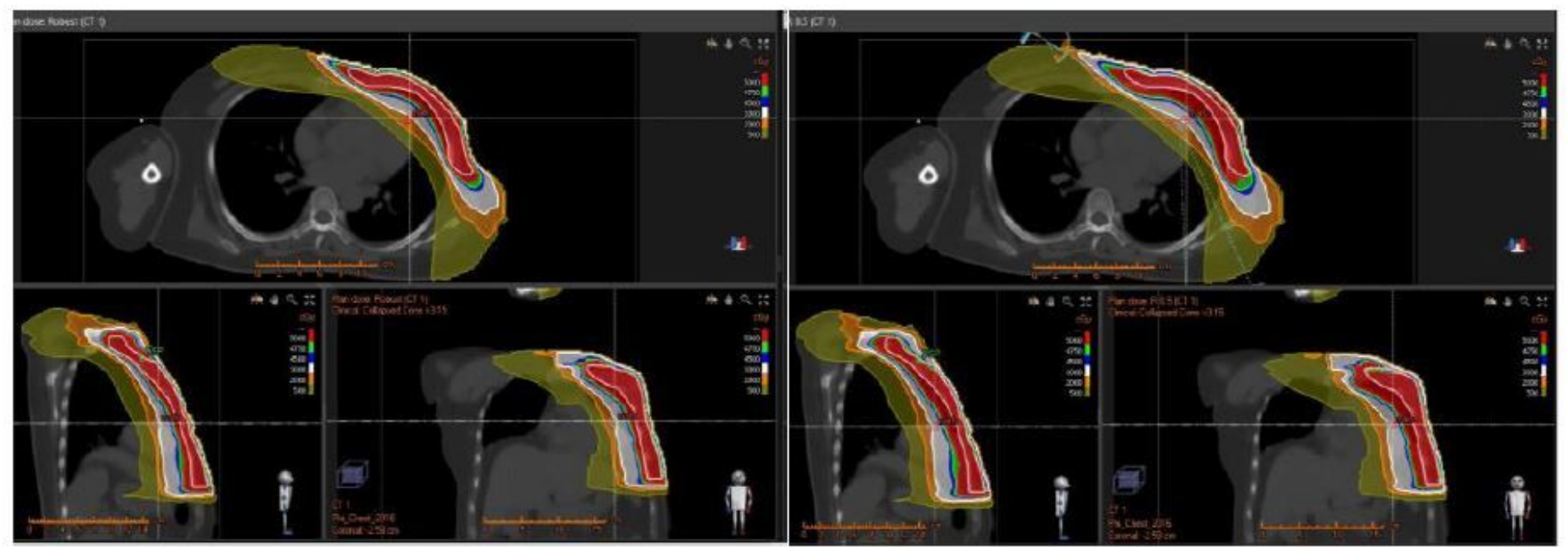

(A)

(B)

Figure 4 
Dose distribution before and after displacement of the center point of optimized radiation field with Robust(A: before shifting;B :after shifting)
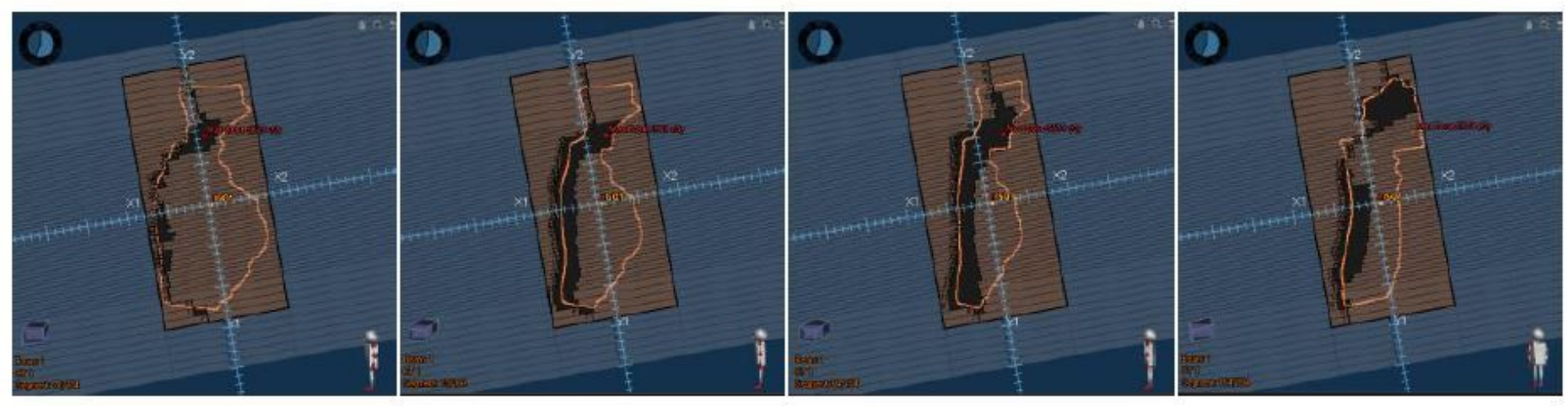

(A)MLC aperture of field direction without Robust optimization
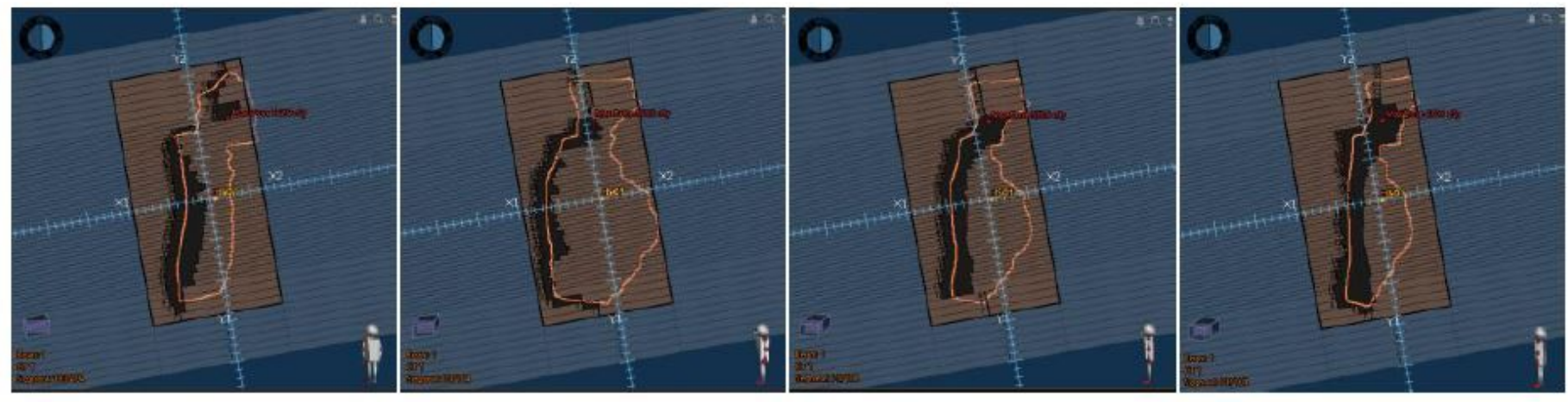

(B)MLC aperture of field direction with Robust optimization

\section{Figure 5}

The comparison of state of Robust optimized multi-leaf collimator A:MLC aperture of field direction without Robust optimization.B:MLC aperture of field direction with Robust optimization.(from left to right, accelerator angle is $76^{\circ}, 96^{\circ}, 118^{\circ}, 148^{\circ}$ in the state of multi-leaf collimator, Robust optimizetion plan was better than no Robust During optimization, blade of multi-leaf collimator has expanded about $0.56 \mathrm{~cm}$ ) 